\title{
O Manejo Psicológico da Dor de Cabeça Tensional
}

Stress headache the psychologycal handling

Resumo: A cefaléia de tensão constitui um dos tipos mais freqüentes de dor de cabeça, embora, etiologicamente, mal compreendida. Ocasiona uma série de comportamentos de evitação que desencadeiam conseqüências físicas, sociais e psicológicas nos indivíduos afetados. O presente trabalho, além de uma revisão da literatura sobre as variáveis contextuais envolvidas nesse transtorno e nas modalidades de tratamento, descreve uma metodologia de investigação capaz de identificar as relações estabelecidas entre o indivíduo, a doença e o meio social. Concluiu-se que uma perspectiva biopsicossocial, integrada a um plano de investigação sustentado por uma formulação comportamental, possibilita uma intervenção mais eficiente, individualizada e sistêmica.

Palavras-Chave: Cefaléia de tensão, formulação comportamental, análise funcional.

Abstract: The tension headache constitutes the kind of headache which happens more frequently, although its ethiology is still not completely understood. It causes an evitation behavior series that unchains physical, social and psychological disturbances. This study has made a critical literature revision, allowing a better

Adriana Mayon N. Flores

Psicóloga. Mestre em Psicologia do Instituto de Psicologia da Universidade de Brasilia

\section{Áderson L. Costa Junior}

Doutor em Psicologia. Professor-adjunto do Instituto de Psicologia da Universidade de Brasilia.

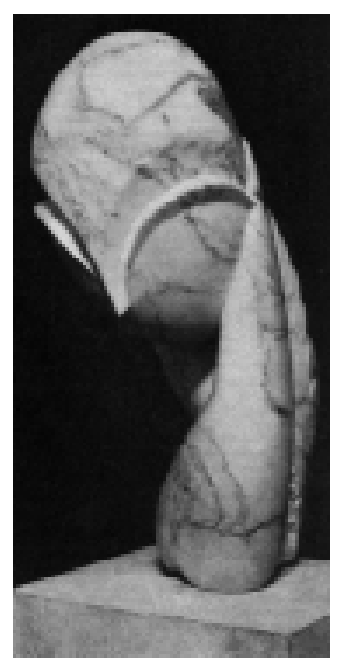
comprehension of this problem and the possible treatments for it. It also intends to develop a methodology for tension headache, able to identify the relationships established among the individual, the disease and the social environment. It concluded that a biopsychosocial perspective, integrated to an investigation plan sustained by a behavioral formulation, is adequate for a more efficient, individualized and systemic intervention

Key Words: Tension headache, behavioral formulation, functional analysis.

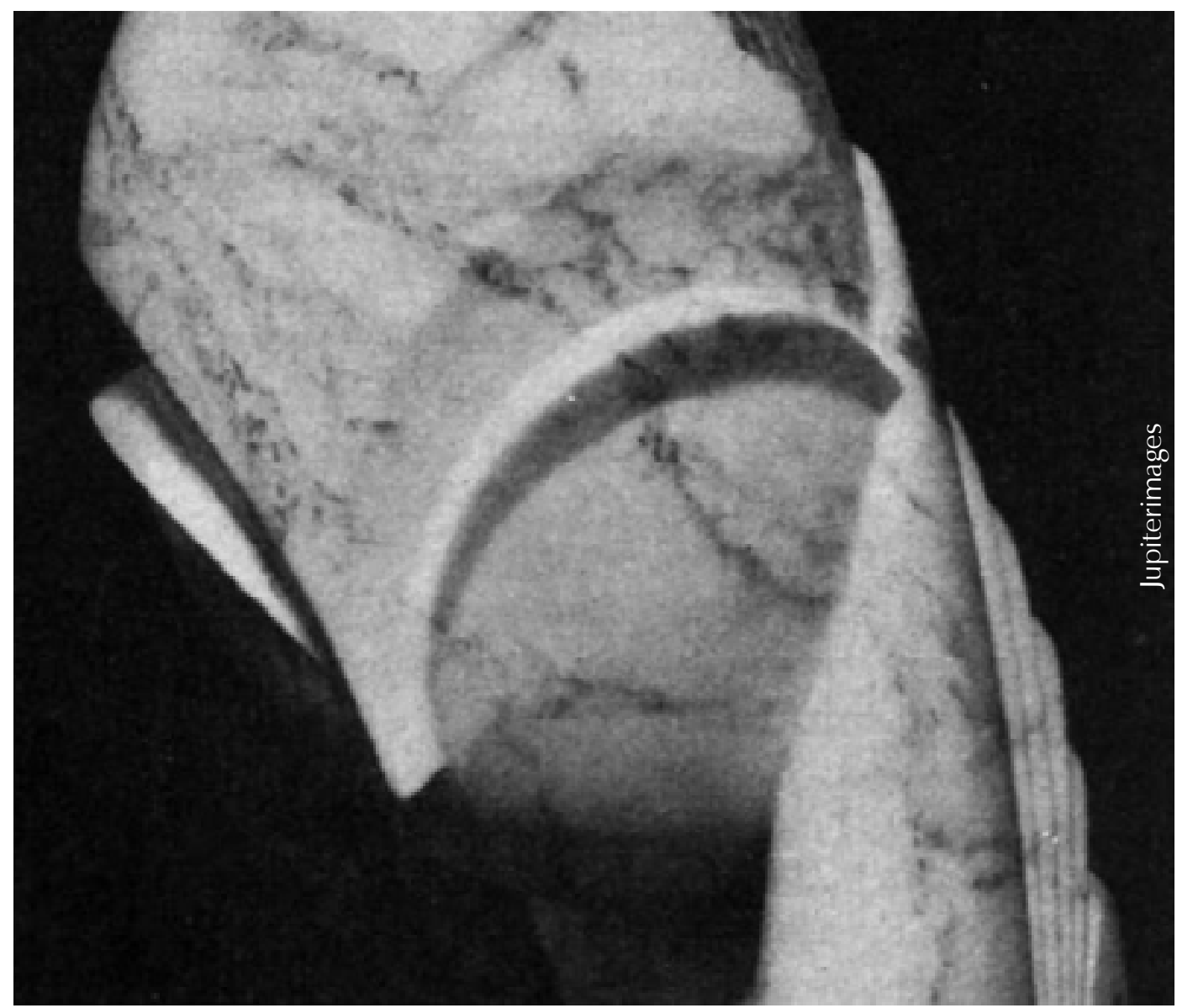


Descrições sobre dores de cabeça ou enxaqueca são encontradas na literatura desde os primórdios da escrita humana. Dentre as diferentes possibilidades de diagnósticos para relatos de dor de cabeça, a cefaléia de tensão é apontada como um dos transtornos mais comuns. Sua dor é insuficientemente compreendida e, freqüentemente, relacionada a fatores emocionais, daí a designação comum de cefaléia de estresse (Adams 1985; Bordini, 2001; Netter, 1986a).

O presente artigo revisa criticamente a literatura sobre cefaléia de tensão e pretende contribuir para a compreensão desse transtorno dentro de uma perspectiva biopsicossocial e interacionista. Em um segundo momento, são apresentadas as modalidades de tratamento mais freqüentemente oferecidas aos pacientes para, finalmente, apontar uma metodologia de investigação potencialmente capaz de apreender a complexidade do tema de acordo com uma proposta sistêmica e idiográfica.

\section{A Cefaléia: Definição e Caracterização}

Bordini (2001) descreve clinicamente a cefaléia de tensão como uma dor bilateral, constritiva e, geralmente, não muito intensa. Ocorre com uma freqüência, em média, menor ou igual a 15 dias por mês (para cefaléia do tipo tensional episódica) ou maior ou igual a 15 dias por mês (para cefaléia do tipo tensional crônica). Náuseas ou vômitos, assim como fotofobia ou fonofobia, estão ausentes, ou apenas uma delas pode estar associada à dor de cabeça. O diagnóstico diferencial nem sempre é preciso, sendo comum o paciente apresentar enxaquecas associadas e referir queixa apenas da dor que é percebida como a mais intensa.

Netter (1986b) alerta para uma seqüência de comportamentos de evitação de desempenhos que fazem parte da vida diária desses pacientes, o que sugeriria quase um padrão. Os relatos revelam que a dor costuma ter início pela manhã e aumentar gradativamente ao longo do dia, podendo persistir durante dias, semanas ou meses. $O$ desempenho em atividades reforçadoras ou o consumo de bebidas alcoólicas pode aliviar os sintomas, fato que diferencia a cefaléia de tensão das enxaquecas.

Em geral, o perfil sociodemográfico do paciente é variável conforme a natureza crônica ou episódica da cefaléia. A primeira tem prevalência menor, sendo mais freqüente em indivíduos do sexo feminino e em populações com menores níveis educacionais. Pode, ainda, aumentar com a idade. Já a cefaléia episódica tem prevalência maior em indivíduos de nível educacional mais elevado, sendo, também, mais comum em mulheres. A faixa etária mais atingida costuma variar dos 20 aos 50 anos, piorando na quarta década e diminuindo com a idade. Relatos médicos alertam para o fato de que pacientes com dor crônica tendem a procurar ajuda profissional com mais freqüência devido à percepção de incômodo persistente e suas conseqüências danosas às condições de enfrentamento do dia-a-dia (Bordini, 2001).

Todavia, segundo Krymchantowski (2001), a maioria dos pacientes com esse tipo de cefaléia não procura ajuda médica no período inicial do problema, sendo comum utilizarem-se de automedicação com analgésicos indicados para outros tipos de dores de cabeça. Há, ainda, o risco do uso abusivo de medicamentos, conduzindo ao agravamento do problema, denominado "efeito rebote", além da possibilidade do desenvolvimento de dependência farmacológica (Holroyd, O'Donnel, Stensaland, Lipchik, Cordingley \& Carlson, 2001).

A fisiopatologia da cefaléia é considerada complexa, sendo possível observar um histórico evolutivo de hipóteses para o problema. Originalmente, foi admitido o papel da contração muscular na etiologia da dor. A contração surgiria como uma reação física a estímulos ambientais ou psicológicos adversos, provocando uma isquemia muscular na nuca e no crânio e produzindo episódios de dor. No entanto, estudos posteriores com eletromiografia não detectaram contração muscular em indivíduos com cefaléia, ficando essa hipótese restrita a uma pequena fração de pacientes com experiência de dor (Bordini, 2001; Rollnik, Karst, Fink \& Dengler, 2001).

Outros estudos revelaram alterações em substâncias endógenas (baixo nível de endorfina no liquor, por exemplo). Isso aumentaria a vulnerabilidade desses pacientes a estímulos ambientais que, normalmente, não produziriam dor, mas passam a ser percebidos como extremamente desagradáveis e potenciais produtores de desconforto físico (Bordini, 2001).

Atualmente, postula-se que a cronicidade da cefaléia de tensão envolva uma sensibilidade neuronal exagerada a estímulos variados, com facilitação ao desenvolvimento da dor. O nervo trigêmio, responsável pela sensibilidade da face e do crânio, recebe toda a informação dolorosa, mas sua interpretação (intensidade e enfoque emocional) depende de outras estruturas (gânglios da base, sistema límbico e núcleos da rafe). Ocorre que, em indivíduos com cefaléia, tais estruturas estariam facilitando a percepção de dor pelo nervo trigêmio. Dessa forma, estímulos físicos, psicológicos e até mesmo a contração muscular seriam percebidos como dolorosos (Bordini, 2001). 
Destaca-se que uma rápida descrição da literatura médica permite apontar uma preferência por explicações que priorizam uma etiologia biológica. As explicações parecem estar relacionadas, filosoficamente, ao modelo biomédico tradicional (Ogden, 1996). Nesse modelo, podemos destacar que a doença é entendida como resultante da exposição do indivíduo, entendido como um agente passivo, a fatores externos de risco. A terapêutica ideal seria aquela que extirparia (ou pelo menos aliviaria) os sintomas incômodos, restaurando o estado de bem-estar do indivíduo.

\section{O modelo sistêmico de saúde traz consigo mudança na forma de compreender o individuo e suas relações como mundo. O indivíduo,} concebido como um ser ativo, atua sobre o meio e assume responsabilidades no processo de adoecer e tratar a doença (Ogden, 1996).

\section{Conformejá apontava Chaves (1982), observa-se uma redução da ênfase sobre a medicina curativa e a adoção de uma perspectiva de incentivo do desenvolvimento de comportamentos para a saúde, denominada "medicina integral".}

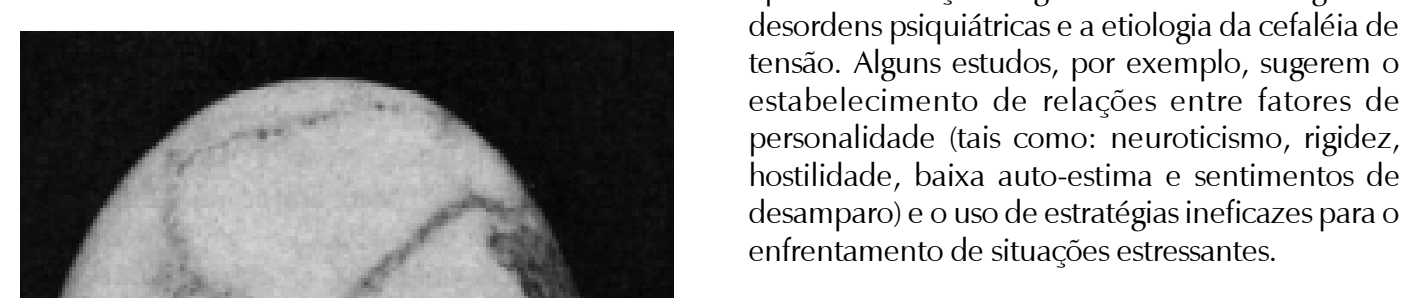

Adams (1985) já apontava a existência de uma tendência, em pacientes com cefaléia, a exibir respostas de dor frente a situações de tensão, como resultado de uma história de modelos familiares desadaptados de enfrentamento ao estresse. Essa tendência manter-se-ia por reforçamento social e por seu valor de esquiva.

Sob a perspectiva da Psicologia da Saúde, percebese que os modelos explicativos mais recentes começam a explorar uma suposta relação funcional entre o indivíduo e a doença. Estariam também incluídos fatores sociais e psicológicos, além daqueles biológicos. Essa tendência de considerar a doença uma condição associada a uma multiplicidade de fatores é coerente com a evolução dos modelos teórico-filosóficos da área de saúde. Se, inicialmente, predominava um discurso biológico, a inserção das ciências humanas no campo da saúde vem possibilitando uma nova concepção da relação mente-corpo, considerando-se a interação do indivíduo com o ambiente sob uma perspectiva mais sistêmica (Martins, 2001; Ogden, 1996).

O modelo sistêmico de saúde traz consigo mudança na forma de compreender o indivíduo e suas relações com o mundo. O indivíduo, concebido como um ser ativo, atua sobre o meio e assume responsabilidades no processo de adoecer e tratar a doença (Ogden, 1996). Conforme já apontava Chaves (1982), observa-se uma redução da ênfase sobre a medicina curativa e a adoção de uma perspectiva de incentivo ao desenvolvimento de comportamentos para a saúde, denominada "medicina integral".

Outra conseqüência desse processo é o desenvolvimento, entre as ciências da saúde, de um novo paradigma biopsicossocial, seguindo uma 
postura integrada que incorpora processos fisiológicos e elementos psíquicos e sociais entre as variáveis que poderiam causar e perpetuar desordens na condição de saúde de indivíduos e grupos (Turk \& Akiko, 2002).

Nesse contexto, segundo Turk e Akiko (2002), qualquer forma de patologia ou mudança física que envolva músculos, juntas ou nervos, gerando informações de dor no cérebro, envolveria, antes de tudo, uma interpretação desse estímulo e formas específicas de se relacionar com ele. Tal processo seria influenciado pelo sistema de crenças do indivíduo, desenvolvido ao longo de sua história de vida. Para Turk e Akiko, a forma de compreender a dor e a enfermidade pode conduzir o indivíduo a uma atitude de ignorar a dor e manter seus compromissos sociais, afetivos e profissionais, ou, por outro lado, abandonar suas responsabilidades e assumir um papel de doente. Uma ampliação desse processo revela fatores sociais no seu background. A escolha de atitude estaria relacionada às reações de outros elementos que reforçam comportamentos saudáveis ou acolhem o indivíduo no papel de doente.

O novo paradigma vem sendo bastante útil em orientar profissionais interessados em otimizar programas de diagnóstico e tratamento para demandas que se desdobram sob dor crônica. A cefaléia de tensão enquadra-se em um desses exemplos, podendo ser abordada de acordo com essa perspectiva (Turk \& Akiko, 2002).

\section{Como os Pacientes Enfrentam a Cefaléia de Tensão}

Considerando a dor como um evento potencialmente estressante, vários estudos buscaram compreender como indivíduos lidavam com os episódios de dor típicos da cefaléia de tensão, incluindo a adoção de estratégias que, ao invés de conduzir a uma melhora da condição de doença, acabavam por contribuir para a sua cronicidade.

Rollnik e colaboradores (2001) compararam a forma como pacientes com cefaléia episódica, cefaléia crônica e indivíduos saudáveis avaliavam e enfrentavam situações de dor. Segundo os autores, os pacientes com cefaléia de tensão episódica tendem a se perceber como capazes de maior controle sobre a dor, não se diferenciando significativamente dos indivíduos normais. Já os pacientes com dor crônica apresentam estados prévios de ansiedade e depressão mais persistentes e um sentimento conseqüente de desamparo, adotando estratégias menos eficazes de enfrentamento da dor. Entre essas estratégias, incluem-se o uso da religião como consolo, a aceitação da dor como um fato consumado, além de respostas de fuga e esquiva, como dormir em horário diurno.

Um aspecto interessante do estudo é que os autores levantam a hipótese de que a adoção contínua de estratégias pouco funcionais para o manejo da dor contribuiria para episódios prolongados de dor, gerando uma redução da qualidade de vida e maior probabilidade de desencadeamento de um quadro clínico de depressão.

Estudos anteriores já apontavam para tais relações. Spinhoven e colaboradores (1991), por exemplo, observaram, em pacientes com cefaléia de tensão, a adoção de estratégias ineficientes de enfrentamento, tais como: (a) negar a dor e seus efeitos; (b) policiar-se (autocontrole) com afirmações do tipo: "todo mundo é capaz de lidar com qualquer tipo de dor", e (c) procurar engajamento em atividades que desviem a atenção da dor. Percebe-se, mais uma vez, uma tendência a comportamentos de fuga e esquiva frente a um estímulo físico e estressor. No mesmo estudo, foi efetuado um levantamento das conseqüências dessas estratégias e observou-se que os pacientes, mesmo quando engajados em atividades concorrentes de distração, experimentavam episódios mais longos de dor durante o dia.

Holm, Lamberty, McSherry e Davis (1997) acrescentaram que indivíduos com cefaléia de tensão tendem a avaliar as situações do dia-a-dia de uma forma mais adversa do que indivíduos saudáveis, o que os leva à escolha de estratégias menos efetivas de enfrentamento. Os autores desenvolveram um estudo que buscava discriminar as características do meio ambiente relacionadas com as reações psicológicas dos sujeitos. Indivíduos com cefaléia de tensão adotam uma visão mais pessimista da realidade, agindo de acordo com essas crenças pessoais.

Os estudos sobre estratégias de enfrentamento em pacientes com dor de cabeça apontam para um perfil de indivíduo que percebe eventos rotineiros como estressores, revelando-se incapaz de reduzir os efeitos adversos por conta própria. Verifica-se uma alta probabilidade de desempenhos típicos de fuga e esquiva associados a uma atitude geral de conformismo, especialmente quando o problema parece fugir ao seu controle, o que aumenta a prevalência de episódios recorrentes de dor crônica.

Estudos que investigam as relações entre transtornos de ansiedade e tensão motora (que pode, em alguns casos, contribuir para a cefaléia de tensão) permitem a compreensão do desenvolvimento de processos crônicos de dor. Segundo Netter 
(1986b), a ansiedade generalizada tem origem na infância, pode agravar-se ao final da adolescência, estando, freqüentemente, associada à depressão e a transtornos de personalidade. Sugere-se que estressores de caráter exógeno costumam agravar a condição ansiogênica, interferindo, substancialmente, sobre a situação de vida desses indivíduos, com prejuízos sociais e ocupacionais.

Observa-se, ainda, que os pacientes com cefaléia de tensão tendem a interpretar os eventos naturais da vida de maneira distorcida. Segundo Netter (1986b), indivíduos ansiosos apresentam maiores dificuldades em parar de se preocupar. São comuns relatos verbais com freqüência, intensidade e duração de preocupação desproporcionais ao impacto real do problema.

Todos esses achados parecem apontar para fatores comuns que circulam pela tendência a exagerar os problemas diários, a preocupar-se de forma excessiva, além da evidente incapacidade em lidar eficientemente com eles. Tal condição afeta diferentes aspectos da vida desses indivíduos, conduzindo a uma gradual redução da qualidade de vida e percepção de piora da doença.

\section{A História do Indivíduo como Variável de Contexto ao Desenvolvimento de Cefaléia de Tensão}

A história de vida do indivíduo contribui, de forma substancial, para a compreensão dos mecanismos de aquisição e manutenção da dor, revelando dados sobre o papel desempenhado pelo contexto ambiental. Estudos sobre variáveis familiares apontam a existência de parentes que também sofriam de dor de cabeça (Adams, 1985; Waldie \& Poulton, 2002), sugerindo uma história de modelação para o comportamento de dor, além de possível herança familiar associada à manifestação do problema.

Segundo Adams (1985), os parentes que percebem suas crianças como "doentes" tendem a mostrarse superprotetores, buscando serviços médicos com mais freqüência. Dessa forma, a família contribui, também, para a manutenção da queixa, uma vez que a criança utiliza a dor como esquiva para situações que envolvam responsabilidades e eventos estressantes, encontrando reforço positivo na atitude dos pais, que a protegem. A conseqüência mais evidente desse processo é a adoção do comportamento de dor como estratégia de enfrentamento diante de situações percebidas como estressantes ou a manutenção da dor decorrente de outros ganhos secundários na vida desses indivíduos.
Entre indivíduos que não relataram histórico familiar de dor, um outro processo vem sendo apontado pela literatura como relacionado etiologicamente à cefaléia por tensão (Adams, 1985; Waldie \& Poulton, 2002). Trata-se de algum trauma sofrido ao longo do desenvolvimento do indivíduo que gerou a primeira crise. Uma vez sanado o problema, a dor persiste, provavelmente, por se tratar de uma oportunidade para o paciente entrar em contato com funções de esquiva e aquisição de reforçamento positivo, tal como descritas anteriormente. Nesses casos, observa-se a presença de reforço social positivo para comportamentos de dependência e manifestações de dor em lugar do encorajamento à autonomia.

O que se pode concluir é que existe uma multiplicidade de fatores atuando sobre o processo de aquisição e manutenção da cefaléia de tensão. O tema inclui explicações biológicas, emocionais e sociais, não sendo possível a identificação de um único determinante. Nesse sentido, seria mais esclarecedor o delineamento de estudos que buscassem identificar as variáveis relevantes a partir de uma perspectiva biopsicossocial, reconhecendose a natureza individual de cada processo patológico. A intervenção mais adequada será aquela capaz de atuar de forma sistêmica, conduzindo ao bem-estar do paciente.

\section{Os Tratamentos Disponíveis para Cefaléia de Tensão}

Em geral, os tratamentos adotados em casos de cefaléia do tipo tensional dividem-se entre os farmacológicos (profilático ou abortivo) e os nãofarmacológicos. Os analgésicos e anti-inflamatórios são comumente utilizados na tentativa de eliminar as crises de dor, e os antidepressivos, como forma de prevenção. Segundo Haas (2001), embora a aplicação local de toxina botulínica também venha sendo empregada na redução dos sintomas de crise, algumas pesquisas apontam que injeções de toxina botulínica, no escalpo e pescoço de pacientes com cefaléia de tensão crônica, não se mostraram mais efetivas, no sentido de aliviar a dor de cabeça, do que injeções de placebo. No entanto, mostraramse eficazes em reduzir a atividade de contração muscular nessas áreas do corpo.

Entre os tratamentos não-farmacológicos, observase uma crescente adoção do biofeedback, conjunto de técnicas que visam a aumentar a habilidade do indivíduo no controle voluntário de respostas físicas, por meio de observação e informações de sinais psicofisiológicos emitidos pelo organismo (Simón, 1996; Donner, 2001), bem como de psicoterapia cognitiva (Adams, 1986; Angelotti, 2001) e intervenções fisioterápicas (Bordini, 2001). 
Netter (1986a) alerta para a possibilidade da existência de transtornos emocionais graves na etiologia da cefaléia de tensão, sendo fundamental a adoção de aconselhamento psiquiátrico. Bordini (2001) faz referência a estudos de neuropsicologia que ajudam os pacientes a conviver, do melhor modo possível, com as crises. Esses trabalhos utilizam técnicas baseadas em conteúdo cognitivo, cujo objetivo é ensinar o paciente a administrar suas crises, sem deixar que a doença o impeça de levar uma vida normal.

Finalmente, Holroyd e colaboradores (2001) compararam grupos de pacientes com cefaléia de tensão crônica que foram submetidos a tratamento farmacológico, grupos que usaram placebo e terapia de controle do estresse e grupos submetidos às duas terapias, farmacológica e psicológica, associadas. Os autores reconhecem maior eficácia quando o tratamento associa elementos farmacológicos e psicológicos ao invés de cada um isoladamente. Haas (2001) defende a adoção de terapias cognitivas e biofeedback, mas lamenta ainda não ter presenciado um único paciente que tivesse apresentado mudanças significativas após ter-se submetido a qualquer uma dessas intervenções.

O que se observa é que o uso de técnicas, farmacológicas ou não, para alívio de sintomas físicos da cefaléia de tensão parece apenas contribuir, temporariamente, para que o paciente retorne às suas atividades quase normais. Seriam necessárias mudanças na forma de o indivíduo se relacionar com o mundo e, conseqüentemente, com a doença, para que os resultados obtidos fossem mantidos por maior período de tempo. Tal proposta requer mudança de concepções sobre o processo de adoecer, considerando tanto dimensões físicas quanto afetivas e sociais.

O tema das cefaléias tensionais parece representar uma demanda em que terapias médicas e de outras ciências da saúde continuam atuando de maneira focal, visando prioritariamente à eliminação de sintomas e apoiando-se em pesquisas que sugerem atuações prescritivas na busca da reabilitação dos pacientes.Inclui-se, ainda, a exigência de resultados epidemiológicos generalizáveis a grandes populações. Tais considerações mostram-se coerentes com a necessidade, apontada pela literatura, da assimilação definitiva do paradigma psicossocial em saúde (Ogden, 1996; Murray \& Chamberlaim, 1999; Cândido, 1998; Morin, 1998).

Estamos, atualmente, em condições de reconhecer o papel fundamental das ciências sociais no campo da saúde, inserindo-se uma nova visão de sujeito e ampliando as perspectivas intervencionistas no campo da prevenção. Houve adesão às metodologias qualitativas propostas pelos cientistas construtivistas e pode-se falar na medicina integral que visa à construção de comportamentos de promoção de saúde. No entanto, permanecem as disparidades entre o avanço tecnológico e as práticas de pesquisa e atuação profissional. Os serviços de saúde colocados à disposição dos pacientes continuam centrados em classificações sintomáticas, rotinas institucionais rígidas e burocráticas e atendimentos distantes do ideal multidisciplinar.

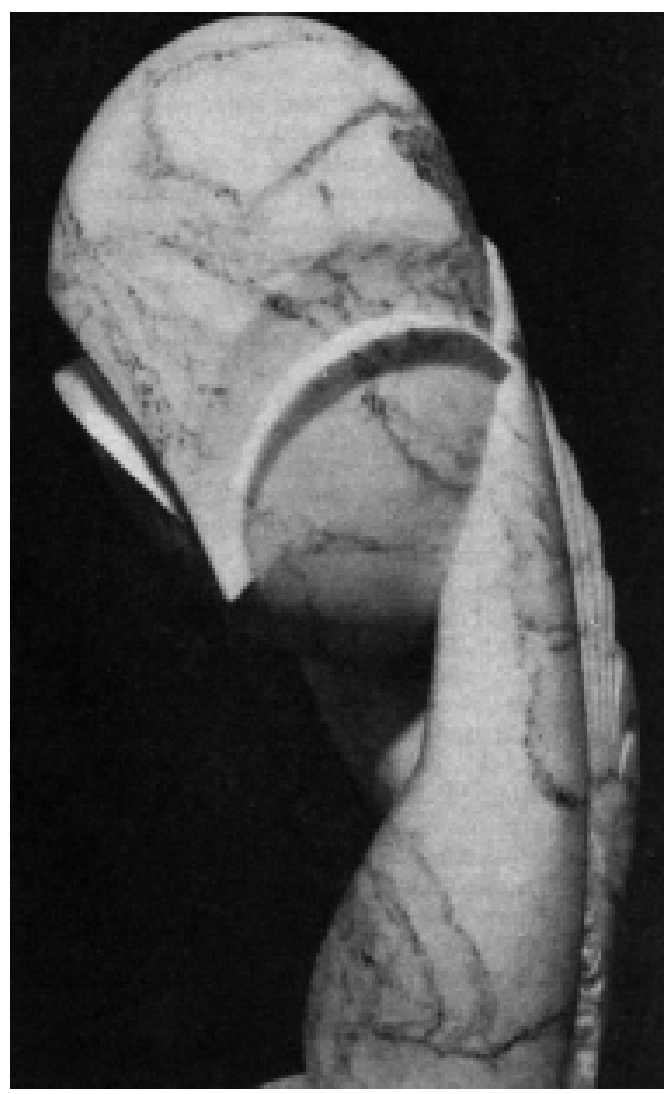

Cândido (1998) lembra que o acesso dos profissionais de saúde a quadros clínicos que envolvam dor crônica sem etiologia definida dá-se, fundamentalmente, pelo relato verbal daqueles que sofrem com o problema. Dessa forma, parece fundamental reconhecer o sentido que a experiência de adoecer ganha na vida dos pacientes. Não se trata, simplesmente, de acolher a queixa e encaminhar o paciente para extensas baterias de exames e/ou tentativas recorrentes de prescrever um medicamento mais eficiente, mas permitir que, ao relatar sua experiência com a dor, o indivíduo seja capaz de ampliar seu conhecimento sobre as relações funcionais que assume com o problema, construindo sua autonomia através do resgate de sua história de vida.

\section{A história de vida do indivíduo contribui, de forma substancial, para a compreensão dos mecanismos de aquisição $e$ manutenção da dor, revelando dados sobre o papel desempenhado pelo contexto ambiental.}


O avanço na compreensão das cefaléias parece condicionado à ampliação de perspectivas para inclusão dos discursos e práticas presentes no atendimento dos usuários do sistema de saúde. Esperar mudanças no comportamento de indivíduos com cefaléia de tensão (enfrentamentos funcionais, incremento na qualidade de vida, maior independência do sistema de saúde, por exemplo) exige mudanças nas práticas que reforçam atitudes de passividade e expectativas irreais de cura.

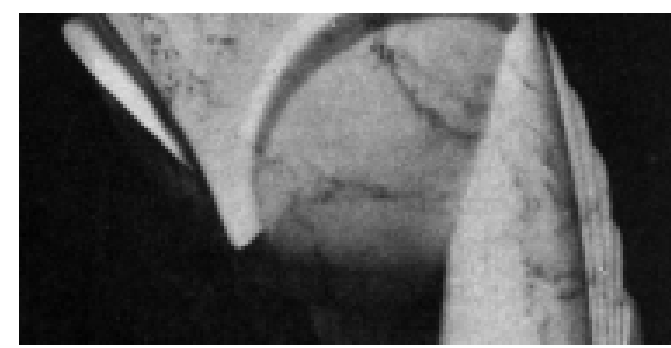

Considerar a doença dentro de um contexto de relações que envolvem o paciente, o meio social e os profissionais de saúde requer uma postura sistêmica do fenômeno e a conseqüente análise das trocas discursivas entre os envolvidos, ressaltando-se que o tempo histórico e a inserção social dos participantes constituem elementos determinantes das representações sobre o adoecer e o curar-se.

\section{A Escolha Metodológica para o Estudo das Cefaléias Tensionais}

A partir dos resultados de estudos que buscam compreender e intervir clinicamente sobre o paciente com cefaléia de tensão, foi possível perceber a existência de padrões típicos de solução de problemas e interações interpessoais que contribuíam para a manutenção da dor e da cadeia comportamental de esquivas, que surgia como desdobramento da experiência cumulativa de dor. Ainda que os estudos se aproximem de perfis de pacientes que sofrem enxaqueca, fica evidente, também, que cada indivíduo reage a estímulos ambientais de forma particular, de acordo com sua própria história de aprendizagem (Wolpe \& Turkat, 1985).

Parece, portanto, impossível abordar um fenômeno humano sem o esforço preliminar de inserir o homem em seu contexto de desenvolvimento, no qual estão em interação sua história de vida e o ambiente atual de determinantes comportamentais. Essa preocupação mostra-se crescente no campo da assistência à saúde (Zannon, 1991). A análise do comportamento vem tentando compreender as experiências humanas de modo a descrevê-las operacionalmente e relacioná-las a fatores circunstanciais. Tal metodologia, denominada de avaliação comportamental, pode ser utilizada tanto com propósitos de tratamento como para delineamentos de pesquisas científicas (Keefe, Kopel \& Gordon, 1980).

Um desdobramento da avaliação comportamental é a formulação de casos que permitam um contato idiográfico e sistêmico com o fenômeno humano que se deseja estudar. Segundo Turkat, Maistro, Burish e Rock (1988), no caso de distúrbios insuficientemente conhecidos, o uso da formulação comportamental constitui um complemento para a pesquisa empírica, no sentido de que vem acompanhada por um suporte de dados que podem ser investigados posteriormente.

A opção pela avaliação comportamental permite a mensuração da linha de base dos comportamentos problemáticos presentes na queixa dos clientes (anterior a qualquer intervenção), bem como a identificação das relações funcionais entre variáveis de controle ou de manutenção e comportamentos problemáticos e, finalmente, a sistematização de objetivos para uma intervenção psicológica mais metódica (Keefe \& cols., 1980)

Para a coleta de dados, são utilizados recursos metodológicos que possam enriquecer a visão do pesquisador sobre o caso. A entrevista visa a facilitar a compreensão da história do problema, seu desenvolvimento e estabilidade ao longo do tempo, bem como flutuações quanto à gravidade e tentativas do cliente de aliviar ou enfrentar (Dengrove, 1979; Keefe \& cols., 1980; Edelstein \& Yoman, 1996). Os questionários auxiliam a obtenção de medidas comportamentais, facilitando a identificação de fatores situacionais associados (Keefe \& cols., 1980). Finalmente, procedimentos de observação direta são úteis em revelar exemplos da ocorrência de comportamentos-problema, podendo ser implementados em situações experimentais ou, quando possível, em situações naturais (Dengrove, 1979).

A formulação comportamental é construída a partir dos dados obtidos em avaliação comportamental e consiste em relacionar os problemas do cliente, fornecendo uma visão sistêmica do caso, capaz de colaborar para a sua compreensão etiológica, além da predição de comportamentos futuros quando o indivíduo estiver exposto a situações semelhantes àquelas estudadas (Turkat \& cols., 1988).

As informações obtidas precisam ser tão completas quanto possível, devendo todo relato relevante ser traduzido em termos de comportamentos, especificando-se seus parâmetros de intensidade, 
freqüência e duração. A etiologia é investigada a partir da reconstrução da história de desenvolvimento do problema, dando ênfase a suas contingências antecedentes e conseqüentes (Keefe \& cols., 1980).

Tal processo de coleta e sistematização de dados biográficos exige o estabelecimento de uma boa relação entre o pesquisador e o entrevistado. A empatia traduzir-se-ia em uma boa compreensão do caso narrado e na capacidade de predizer comportamentos futuros do indivíduo. Tudo isso só vai ser possível a partir da habilidade do profissional em criar um ambiente facilitador de auto-expressões (Wolpe \& Turkat, 1985).

Nos casos em que não se verifica a correspondência entre comportamentos verbais e ações, ou onde a ambigüidade gera dificuldades na formulação de hipóteses, cabe ao pesquisador refazer sua linha de questionamento, buscando outros exemplos associados. Assim, seria obtida uma visão das práticas do indivíduo para, então, relacioná-las ao que já foi relatado (Turkat, 1985).

\section{Um Esboço das Etapas da Formulação Comportamental}

Segundo Wolpe \& Turkat (1985), o procedimento de reformulação comportamental deve ser conduzido com o objetivo de coletar informações pertinentes às seguintes temáticas:

Que problemas o indivíduo vem experienciando no dia-a-dia?

Quais são os problemas comportamentais?

Quais as relações funcionais entre o ambiente e o repertório de comportamentos?

Por que essas relações funcionais se desenvolveram e persistiram?

Que fatores poderiam produzir mudanças comportamentais significativas?

A partir desses dados, o pesquisador pode iniciar a formulação de hipóteses, sendo importante que inicie tal atividade o mais cedo possível, de modo a conduzir suas perguntas no sentido de testar as hipóteses.

Dicas sobre o entrevistado podem ser obtidas na própria observação de sua apresentação clínica e atitudes ao longo do primeiro encontro. $\mathrm{O}$ registro deve incluir hipóteses que relacionem essas pistas (Dengrove, 1979). A queixa também pode funcionar, nesse sentido, por expor as minúcias do problema, assim como seus fatores mantenedores e outros problemas relacionados (Wolpe \& Turkat, 1985).

A entrevista deve ser conduzida buscando uma melhor identificação e mensuração de comportamentos-problema. Ao final do processo, o pesquisador deve ser capaz de reescrever a história que lhe foi narrada, mantendo a coerência entre os fatores mantenedores atuais e aqueles que atuaram na aquisição do problema. Enfatizam-se os aspectos cronológicos e contextuais (circunstâncias para a ocorrência e conseqüências na vida do indivíduo), mantendo o interesse adicional em investigar outros comportamentos associados ao problema, tais como reações físicas e cognições.

Uma vez que o pesquisador já tenha coletado o depoimento autobiográfico do entrevistado e reproduzido esse depoimento em forma de texto, salientando os aspectos relevantes a uma avaliação comportamental, resta, ainda, formular hipóteses sobre as condições precipitadoras dos comportamentos problemáticos (Wolpe \& Turkat, 1985). O pesquisador deve buscar associar a narrativa do sujeito a uma série de hipóteses que poderão ajudar na escolha de objetivos para intervenção, daí seu caráter diagnóstico e individual.

A etapa seguinte inclui uma devolução da formulação, de maneira que o cliente compreenda como seus problemas foram percebidos. Assim, ele obtém maior autonomia no sentido de concordar ou discordar e modificar sua história. A discussão sobre modalidades terapêuticas constitui uma excelente estratégia para compartilhar o processo de tomada de decisão (Wolpe \& Turkat, 1985). Os autores reconhecem a necessidade de se reduzir a quantidade de informações técnicas. Agindo dessa forma, o pesquisador aumenta a possibilidade de compartilhar significados em comum com o cliente, ampliando seu nível de confiança e motivação para a mudança comportamental.

\section{Considerações Finais}

Dada a complexidade do fenômeno das cefaléias tensionais e o fato de este ser um problema de etiologia ainda insuficientemente esclarecido, parece muito importante cuidar para que o tratamento proposto não pressuponha um perfil único de paciente, nem uma única forma de manifestação biológica da doença, mas considere as individualidades de cada caso, assumindo um caráter dinâmico e sujeito a reavaliações periódicas. A proposta da formulação comportamental parece bastante adequada a tais objetivos.
Dada a complexidade do fenômeno das cefaléias tensionais e o fato de este ser um problema de etiologia ainda insuficientemente esclarecido, parece muito importante cuidar para que o tratamento proposto não pressuponha um perfil único de paciente, nem uma única forma de manifestação biológica da doença, mas considere as individualidades de cada caso, assumindo um caráter dinâmico e sujeito a reavaliações periódicas. 


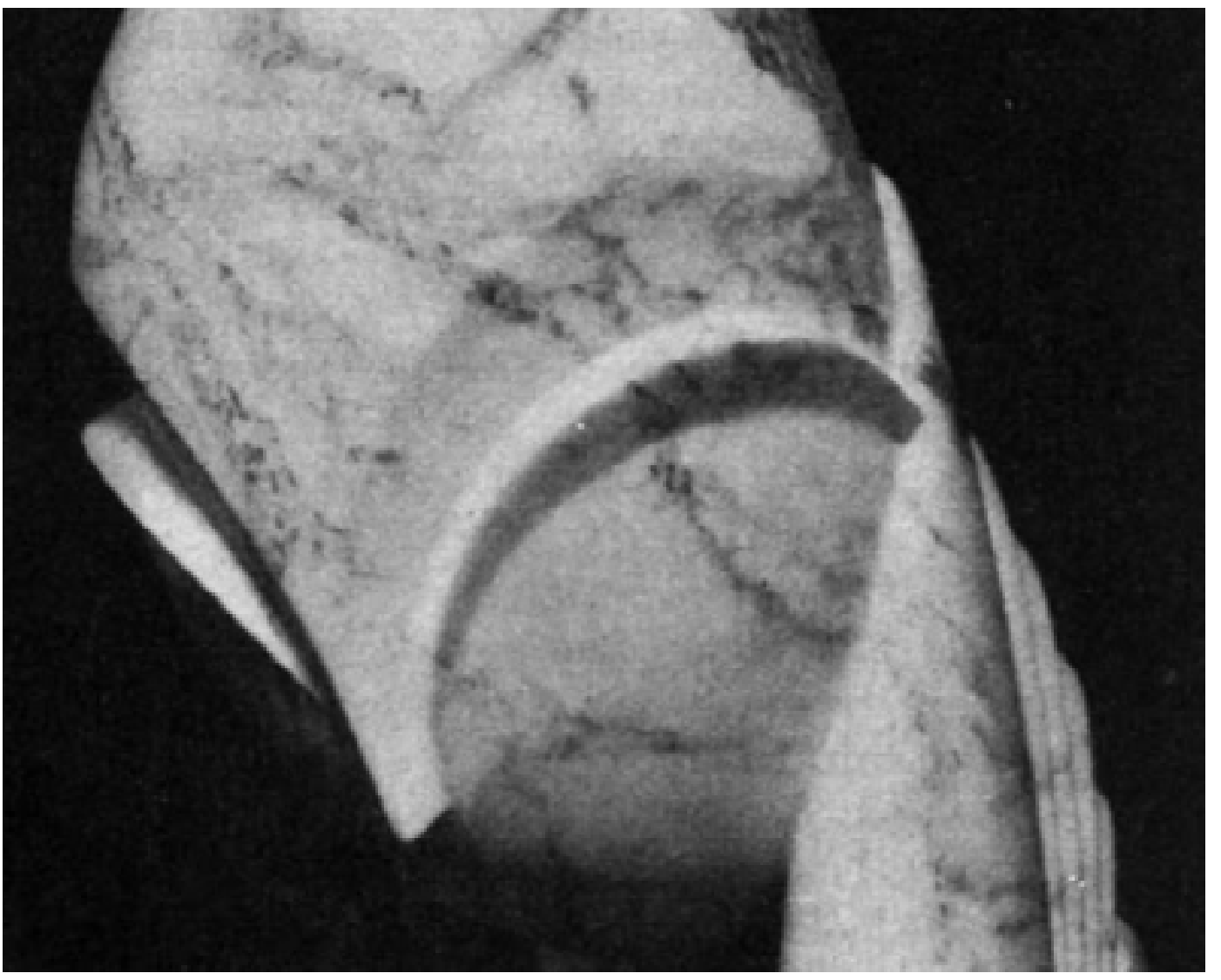


ANGELOTTI, G. Tratamento da Dor Crônica. In B. Range (org.). Psicoterapias Cognitivo-comportamentais. Porto Alegre: Artmed, 2001, pp. 535-545.

ADAMS, H.E. Case Formulations of Chronic Headaches. In J. D. Turkat (org.). Behavioral Case Formulation. New York: Plenum Press, 1985, pp.87-108.

BORDINI, C.A. Cefaléia do Tipo Tensional. In C.A. Bordini \& Cols. (orgs.). Retratos da Enxaqueca e das Cefaléias Primárias. São Paulo: Lemos, 2001, pp. 59-64.

CÂNDIDO, C.L. Sobre o Paciente com Dor Crônica: uma Perspectiva Psicossocial do Processo de Adoecimento. Dissertação de Mestrado, Universidade de Brasília, Brasília, 1998.

CHAVES, M. Saúde e Sistemas. Rio de Janeiro: Guanabara, 1982. DONNER, I.O. Biofeedback. In B. Range (org.). Psicoterapias Cognitivocomportamentais . Porto Alegre: Artmed, 2001, pp.131-142.

DENGROVE, E. Diagnóstico Comportamental. In A. A. Lazarus (org.). Terapia Comportamental na Clínica. Belo Horizonte: Interlivros, 1979.

EDELSTEIN, B.A. \& YOMAN, J. A Entrevista Comportamental. In V. E. Caballo (org). Manual de Técnicas de Terapia e Modificação do Comportamento. São Paulo: Livraria Santos Editora, 1996.

HAAS, D.C. Treatment of Episodic and Chronic Tension-type Headaches. In http://www.upstate.edu/neurology/haas/index.html. Suny Upstate Medical University, 2001

HOLM, J.E., LAMBERTY, K., MCSHERRY, W.C. \& DAVIS, P.A. The Stress Response in Headache Sufferers: Physiological and Psychological Reactivity. Headache, 37, pp. 221-227.

HOLROYD, K.A., O'DONNEL, F..D.O., STENSLAND, M., LIPCHIK, G.L., CORDINGLEY, G.E. \& CARLSON, B.W. Management of Chronic Tension-type Headache with Tricyclic Antidepressant Medication, Stress Management Therapy, and their Combination: a Randomized Controlled Trial. The Journal of the American Medical Association, 285, 2001, pp. 2208-2215.

KEEFE, F.., KOPEL, S.A. \& GORDON, S.B. Manual Prático de Avaliação Comportamental. São Paulo: Manole, 1980.

KRYMCHANTOWSKI, A.V. Cefaléia. Revista Brasileira de Medicina, 58, 2001, pp. 59-79.

MARTINS, B.M. A Comunicação no Contexto de Reabilitação: o Encontro Entre Enfermeiro e Paciente em uma Unidade de Assistência aos Lesados Medulares. Dissertação de Mestrado, Universidade de Brasília, Brasilia, 2001.

MORIN, E. Ciência com Consciência. Rio de Janeiro: Bertrand, 1998. MURRAY, M. \& CHAMBERLAIM, K. Qualitative Health Psychology: Theories \& Methods. London: Sage, 1999.
NETTER, F.H. Muscle Contraction Headache. In F.H. Netter (org.). The Ciba Collection of Medical Illustrations N. York: CIBA-GEIGY Corporation, 1986a, pp.32-33

Common Problems in Psychiatry. In F.H. Netter (org.). The Ciba Collection of Medical Illustrations. N. York: CIBA-GEIGY Corporation,1986b, pp.136-141.

OGDEN, J. Health Psychology - a Textbook. Buckingham: Open University Press, 1996.

ROLLNIK, JD., KARST, M., FINK, M. \& DENGLER, R. Coping Strategies in Episodic and Chronic Tension-type Headache. Headache, 41, 2001, pp. 297-302.

SIMÓN, M.A. Biofeedback. In V.E. Caballo (org.). Manual de Técnicas de Terapia e Modificação do Comportamento. São Paulo: EditoraSantos, 1996, pp.335-358.

SPINHOVEN, P., JOCHEMS, P.A., LINSSEN, A.C. \& BOGAARDS, M. The Relationship of Personality Variables and Patient Recruitment to Pain Coping Strategies and Psychological Distress in Tension Headache Patients. Clinical Journal of Pain. 7, 1991, pp.12-20.

TURK, D.C. \& AKIKO, O. Psychological Factors in Chronic Pain: Evolution and Revolution. Journal of Consulting and Clinical Psychology, 70 (3), 2002, pp. 678-690.

TURKAT, I.D. Behavioral Case Formulation. New York: Plenum Press, 1985

TURKAT, I.D., MAISTRO, S.A., BURISH, T.G. \& ROCK, D.L. Verificação da Formulação Sobre Casos Clínicos. In H.W. Lettner \& B.P. Rangé (orgs). Manual de Psicoterapia Comportamental. São Paulo: Manole, 1988.

WALDIE, K.E. \& POULTON, R. Physical and Psychological Correlates of Primary Headache in Young Adulthood: a 26 Year Longitudinal Study. Journal of Neurology, Neurosurgery \& Psychiatry, 72, 2002, pp. 86-92.

WELCH, K.M.A. A 47-year-old Woman with Tension-type Headaches. The Journal of the American Medical Association, 286, 2001, pp.960-966.

WOLPE, J. \& TURKAT, J. D. Behavioral Formulation of Clinical Cases. In J.D. Turkat, (org.). Behavioral Case Formulation. New York: Plenum Press, 1985, pp. 5-35.

ZANNON, C.M.L.C. Desenvolvimento Psicológico da Criança: Questões Básicas Relevantes à Intervenção Comportamental no Ambiente Hospitalar. Psicologia: Teoria e Pesquisa, 07, 1991, pp.119-136. 